\title{
Selected features of morpho-functional reactions of eukaryotic microorganisms grown in the presence of maghemite iron oxide nanoparticles obtained by laser target evaporation
}

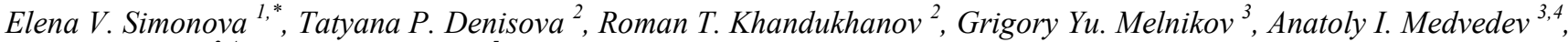 \\ Igor V. Beketov ${ }^{3,4}$, Ricardo Andrade. ${ }^{5}$ \\ ${ }^{1}$ FSBEI HE Irkutsk State Medical University MOH, Red Revolt str., 1, Irkutsk, 664003, Russia \\ ${ }^{2}$ Irkutsk State University, Nizhnyaya Embankment St., 6, Irkutsk, 664011, Russia \\ ${ }^{3}$ Ural Federal University, Mira St. 19 Ekaterinburg, 620000, Russia \\ ${ }^{4}$ Institute of Electrophysics, Amundsen str., 106, Ekaterinburg, 620016, Russia \\ ${ }^{5}$ Universidad del País Vasco UPV-EHU, Leioa, 48940 Spain
}

\begin{abstract}
Biological activity of iron introduced into nutrient medium as a suspension of iron oxide nanoparticles (MNPs) synthesized by the laser target evaporation was investigated. Exophiala nigrum (E. nigrum) eukaryotes were grown either in the presence or in absence of MNPs. De-aggregated suspensions of $\mathrm{Fe}_{2.75} \mathrm{O}_{4}$ MNPs were added in concentrations of 1 to $10^{4}$ maximum permissive dose (MPD, being 0.3 $\mathrm{mg} / \mathrm{L}$ of Fe ions in water). Cells were exposed for 24 to 96 hours periods and then plated onto a solid medium. The effect of MNPs was evaluated by the change in the number of cells during exposure and the number and morphology of the colonies. For 1-10 MPD yeast showed unaltered characteristics. For 100 or 1000 MPD for 72 hours of exposure and above the number of cells increases up to 30 times in comparison with the control. A pronounced stimulating effect was revealed at $10^{4}$ MPD of iron. A significant excess of the number of cells was observed for the first day. At exposures of 72 and 96 hours the differences in the number of cells in comparison with the control were 11 and 30 times, respectively. A change in the colonies morphology was observed at 100 MPD concentration.
\end{abstract}

\section{Introduction}

Recent focus on wide employment of magnetic nanoparticles (MNPs) in wide spectrum of biomedical applications [1-2] requires the study of the features of their interactions with biocomponents, in particular with eukaryotic organisms. Iron oxides such as magnetite $\left(\mathrm{Fe}_{3} \mathrm{O}_{4}\right)$ and maghemite $\left(\gamma-\mathrm{Fe}_{2} \mathrm{O}_{3}\right)$ are well known as materials with a high degree of biocompatibility, a sufficiently high saturation magnetization at room temperature and a high Curie temperature [3]. Physical and chemical properties of their nanosized analogues are less studied, especially in the case of assessments of their cytotoxicity and environmental impact [4-5]. Biological applications require the most complete attestation of MNPs by various methods [6-7], since even the most insignificant changes in the conditions of production can lead to a change in their functional properties. Nanoparticles of iron oxides with very small nonstoichiometry of the composition are able to act as catalysts and introduce additional peculiarities to the morpho-functional reactions of the living systems. In addition, for pharmaceutical products containing nanocomponents, there is a requirement for additional testing of samples of each lot just prior to application of the drug [7]. These circumstances are the reason that the toxic, cytotoxic and genetic effects of MNPs require further study and refinement. The understanding of the interaction between the living system and magnetic nanoparticles and possibility of incorporation of MNPs with reasonably large magnetic moment open new research and clinical opportunities $[4,8]$.

Exophiala nigrum (E. nigrum, black unicelular eukaryote yeast) playing an important role in the equilibrium of ecosystem of the Baykal Lake [2] can be used as a model system biological experiments with MNPs.

This study was focused on the morpho-functional reactions of $E$. nigrum eukaryotic microorganisms in response to the effect of $\mathrm{Fe}_{2.75} \mathrm{O}_{4}$ iron oxide magnetic nanoparticles in form of electrostatically stabilized water-based suspension.

\section{Experimental}

For the fabrication of spherical iron oxide magnetic nanoparticles an electrophysical technique of the laser target evaporation (LTE) was employed [2]. Compacted commercial $\mathrm{Fe}_{2} \mathrm{O}_{3}$ powder target (Alfa Aesar) was used in the LTE installation designed and developed at the 
Laboratory of pulsed processes of the Institute of Electro physics UD RAS. The target was overheated by the high-energy pulse of focused laser beam of ytterbiumdoped fibre laser (1.07 $\mu \mathrm{m}$ wavelength). More details on LTE technique can be found elsewhere [2,9].

Structure of LTE MNPs was studied by transmission electron microscopy (TEM, JEOL JEM2100 microscope operating at $200 \mathrm{kV}$ ) and the X-ray diffraction (XRD) (DISCOVER D8, Bruker diffractometer using $\mathrm{Cu}-\mathrm{K} \alpha$ radiation). The stoichiometric ratio of $\mathrm{Fe}^{2+} / \mathrm{Fe}^{3+}$ in the iron oxide MNPs was determined based on the XRD analysis and redox potentiometric titration using a TitroLine titrator. Suspensions of LTE MNPs in distilled water electrostatically stabilized by the sodium citrate ( 5 $\mathrm{mM}$ ) were prepared by ultrasound treatment (ColePalmer CPX750). Hermle Z383 centrifuge operated at $10000 \mathrm{rpm}$ for $10 \mathrm{~min}$ was used to remove large aggregates. Magnetic measurements were carried out on a MPMS XL-7 SQUID-magnetometer.

As a test culture, melanin-containing microscopic fungi Exophiala nigrum, was isolated from the natural population of Baikal Lake. Toxic properties of a suspension of iron oxide MNPs synthesized by laser evaporation were studied for E. nigrum, grown in the presence or absence of (control samples) MNPs. E. nigrum has a black colour due to the produced melanin [10-11]. Primary concentration of MNPs in suspension was $5 \%$ by weight. Suspensions of $\mathrm{Fe}_{2.75} \mathrm{O}_{4}$ MNPs solutions for biological experiments were prepared on the basis of Saburo liquid nutrient medium in concentrations $1-10^{4}$ MPD. According to the sanitary and hygienic norms, 1 MPD is $0.3 \mathrm{mg} / 1$ of $\mathrm{Fe}$ ions in water, but the determination of the maximum allowable concentration refers to soluble forms of iron [11].

The control was Saburo liquid medium with the corresponding addition of a sterile physiological solution without MNPs. In all cases, $1 \mathrm{ml}$ of E. nigrum cell suspension was added. Flasks with sowings were placed on a mechanical rocking chair. Exposure times were 24, 48,72 or 96 hours. At the end of each exposure period, cultures were planted onto a solid nutrient medium (agar Saburo). Considered indicators were: the number of cells at the beginning of the experiment and after exposure (measured in the Goriaev's chamber), the number and morphology of the colonies grown on a solid nutrient medium [12].

For TEM studies of biological samples cell cultures were pre-fixed in $0.5 \%$ glutaraldehyde in Sörenson buffer and centrifuged. The supernatant was removed and 2\% glutaraldehyde in Sörenson buffer was added to the pellet. Samples were fixed in $1 \%$ osmium tetroxide in Sörenson buffer, dehydrated, embedded in EponPolarbed resin and cut in ultrathin sections for TEM studies (Philips EM208S). More details on the technique can be found elsewhere [4].

\section{Results and discussion}

Figure 1(a) shows a TEM micrograph of the MNPs of iron oxide: the shape of the nanoparticles is close to spherical. For this batch the size distribution calculated

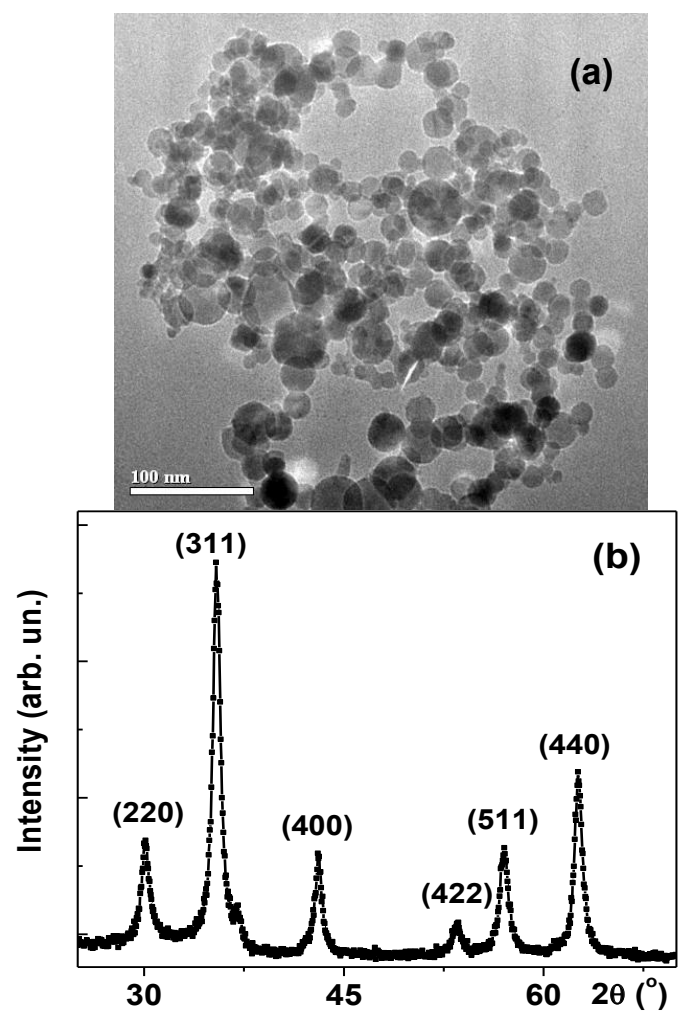

Fig. 1. TEM image of LTE MNPs (a). XRD patterns of iron oxide MNPs. Miller indexes is given in round brackets (b).

from the TEM images of the MNPs was lognormal. Figure 1(b) shows the X-ray spectrum of the MNPs of iron oxide. The experimental points for studied MNPs are fitted well by the magnetite XRD database. However, it is well known that the magnetite $\left(\mathrm{Fe}_{3} \mathrm{O}_{4}\right)$ structure can not be distinguished from that of maghemite $\left(\gamma-\mathrm{Fe}_{2} \mathrm{O}_{3}\right)$ based only on XRD data [3]. The crystalline cells of these oxides correspond to the inverse spinel structure (Fd3m space group). In the present study, the stoichiometric ratio $\mathrm{Fe}^{2+} / \mathrm{Fe}^{3+}$, determined from the analysis of the interplanar distances of the crystal lattice and the potentiometric titration data, was $\mathrm{Fe}_{2.75} \mathrm{O}_{4}$.

The number-average $\left(D_{N}\right)$ and mass median $\left(D_{W}\right)$ diameters [13] were measured using the graphical analysis of TEM images and the statistical sampling of not less than 2000 individual particles. It should be specially noted that the mean values calculated using various techniques (TEM, XRF) correlate very well with each other $D_{\text {XRD }}=23 \pm 3 \mathrm{~nm}$ (for XRD), $D_{N}=23 \pm 3 \mathrm{~nm}$ and $D_{W}=34 \pm 4 \mathrm{~nm}$. The size of the coherent scattering region $\mathrm{D}_{\mathrm{XRD}}$ was determined by the Scherrer method based on the XRD patterns [14].

The hysteresis loop of as-prepared MNPs (not shown here) indicates no saturation at room temperature and very low coercivity: the magnetization in the field of $65 \mathrm{kOe}$ was close to $55 \mathrm{emu} / \mathrm{g}$. The comparative analysis of structural features and magnetic parameters confirms consistency of obtained data about close to superparamegnetic behaviour for MNPs of this size. Reasonably high value of the magnetic moment of each superparamagnetic nanoparticle makes this kind of materials promissing for such biomedical applications as magnetic hyperthermia or thermal ablation, magnetic 
Table1. Number of cells in $1 \mathrm{ml}$ of test solutions

\begin{tabular}{|c|r|r|l|l|l|l|}
\hline \multirow{2}{*}{$\begin{array}{c}\text { Exposure, } \\
\text { hours }\end{array}$} & \multicolumn{6}{|c|}{ Number of cells in 1 ml, $\times \mathbf{1 0}^{\mathbf{6}}$} \\
\cline { 2 - 7 } & $\begin{array}{c}\text { MPD } \\
\text { MPPD }\end{array}$ & \multicolumn{1}{|c|}{ MPD } & \multicolumn{1}{c|}{$\begin{array}{l}\text { MPD } \\
\text { MPD }\end{array}$} & $\begin{array}{l}10^{3} \\
\text { MPD }\end{array}$ \\
\hline 0 & 2.0 & 2.2 & 1.6 & 2.0 & 2.6 & 2.0 \\
\hline 24 & 10.6 & 14.6 & 18.0 & 15.2 & 13.2 & 546.0 \\
\hline 48 & 13.4 & 17.0 & 13.2 & 15.2 & 15.0 & 584.0 \\
\hline 72 & 10.6 & 11.4 & 6.8 & 30.0 & 43.0 & 116.0 \\
\hline 96 & 7.6 & 15.4 & 9.4 & 35.0 & 230.0 & 228.0 \\
\hline
\end{tabular}

Field assisted drugs delivery and magnetic biosensing $[4,8]$.

The maximum iron oxide concentration of the electrostatically stabilized aqueous suspensions was 37 $\mathrm{g} / 1$ with an average hydrodynamic aggregate size of 86 $\mathrm{nm}$ and an averaged value of the zeta potential (-42.5) $\mathrm{mV}$. A detailed analysis of the structure of the MNPs and the parameters of aqueous suspensions allows accurate calculation of the iron content in media for the biological part of the studies. But we must mention the existing ambiguity in determining the maximum permissible dose in the case of MNPs. Nanoparticles and, in a broader sense, colloidal particles, strictly speaking, can not be considered a soluble form. The particles are a separate insoluble phase with an interface with the aqueous medium. Although some iron solubility on the boundary of the particle is, but most likely it does not exceed one MPD [15].

Figure 2 shows examples of TEM typical morphology of Exophiala nigrum cells grown without and with iron oxide MNPs. The pronounced inclusion of nanoparticles into the cells was not observed. Counting the number of cells in the Goriaev's chamber was carried out in 20 squares, and then the average value of the number of cells in $1 \mathrm{ml}$ was determined from the known formulas [11]. The results are shown in Table 1. It is important to mention that at high iron concentrations of 1000 and 10000 MPDs intensive yeast growth was noted, which resulted in a very high cell density in $1 \mathrm{ml}$ : from 228 to $584\left(\times 10^{6}\right)$. Figure 3 shows the change in the number of cells in $1 \mathrm{ml}$ of test solutions, depending on the exposure period. Considering the high concentration of cells, the $\log$ of cell number per $\mathrm{ml}$ is shown on the ordinate axis. Thus, suspensions of iron oxide nanoparticles in the concentrations of 1 or 10 MPD do not have an active stimulating effect for Exophiala nigrum yeast cells, in contrast to 100, 1000 and 10000 MPD. It was found that the higher the concentration of MNPs in the suspension of nanoparticles, the stronger the stimulating effect, expressed in the high activity of yeast cell multiplication.

Additions of a suspension in iron concentrations of 100 and 1000 MPD provided by MNPs of the iron oxide starting from 72 hours of exposure resulted in the increase of the number of Exophiala nigrum cells from 3-5 to30 times in comparison with the control $(\mathrm{P}<0.05)$. For a suspension of MNPs at a concentration of $10^{4}$ MPD of iron a significant excess (52 times) of the num-
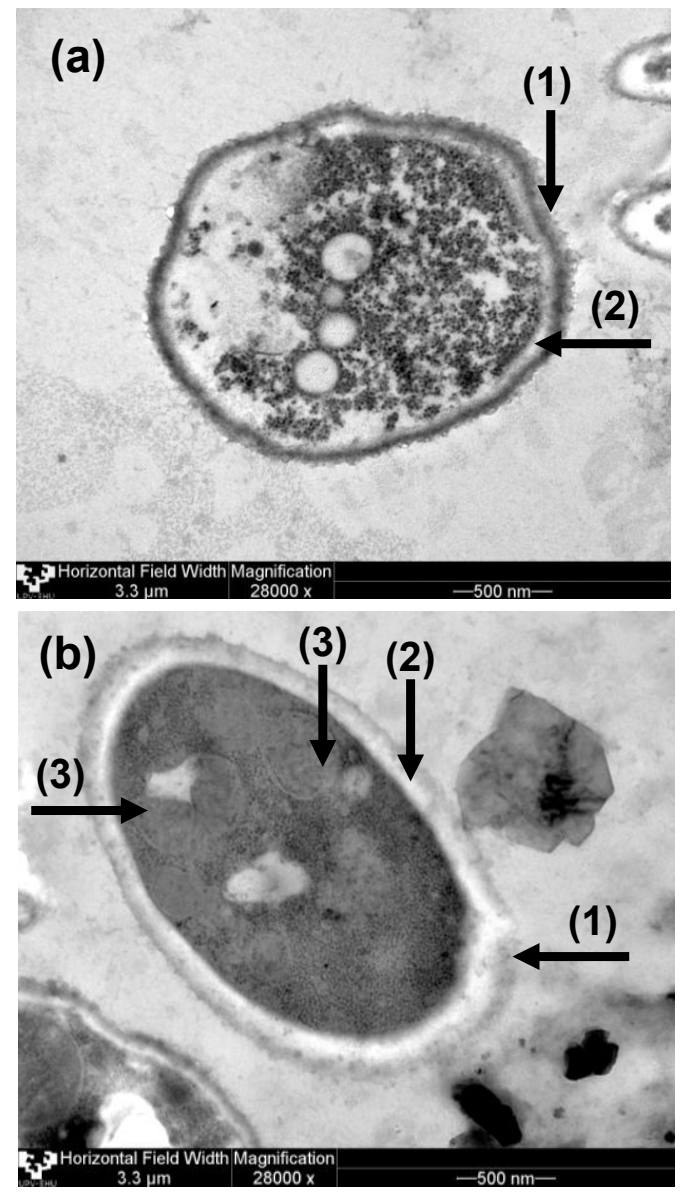

Fig. 2. TEM image of E. Nigrum cell grown without MNPs (control) (a) and in the presence of $10^{\circ} \mathrm{MPD}$ of iron provided by citric acid stabilized MNPs from suspension (b). Cell wall (1), a periplasmic space (2) and mitochondria (3) of black yeast.

ber of cells in $1 \mathrm{ml}$ compared with the control was observed in the first day of exposure. At exposures of 72 and 96 hours, the difference with the number of cells in the control variant is 11 and 30 times, respectively (P $<0.05)$.

Naturally, a high density of cells in $1 \mathrm{ml}$, led to the fact that a large number of colonies grew onto solid substrates in Petri dishes. Moreover, after 48 hours of exposure, counting colonies in Petri dishes proved to make no sense, because the growth of yeast reached the saturation level. Suspensions of MNPs in concentrations of 100 and 1000 MPD are moderately toxic, and at a concentration of $10000 \mathrm{MPC}$ - are toxic.

Interesting results were obtained in an experiment with MNPs suspension in a concentration of 100 MPD for biological experiments. At the beginning of the exposure, as well as after exposures at 24 and 48 hours, all grown up colonies on a solid substrate visually had a typical morphology for E. nigrum: black color. After exhibiting the yeast in this suspension for 72 hours, on a solid substrate against a background of a set of typical black colonies of E. nigrum unusual single white colonies grew. After the exposure for 96 hours, colonies of white colour predominated on a solid substrate. It should be noted that very similar pattern was observed in all replicates of the same experiment. 


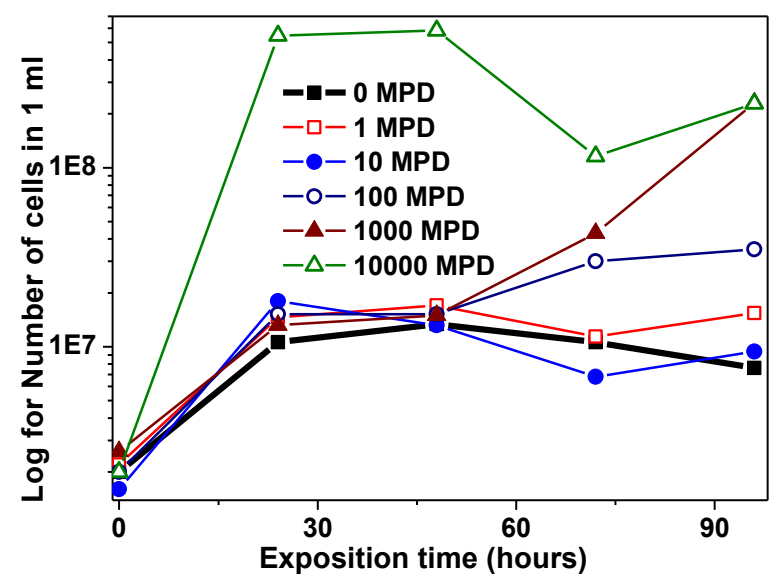

Fig. 3. Change in the concentration of Exophiala nigrum yeast cells in $1 \mathrm{ml}$, depending on the exposure time in different for different iron concentrations measured in maximum permissive doses.

The results obtained in the present work require further development for better understanding of the mechanism of stimulation of cell divisions of E. nigrum yeasts and revealing the nature of the heterogeneity of the population according to the morphological criteria induced by the presence of MNPs. We suggest that $E$. nigrum cages contain (or adhere on the surface of the membrain) iron oxide in the form of MNPs [2, 10,16], which makes yeast an attractive model object at the stage of development of suspensions for hyperthermia, thermal ablation and magnetic biosensing.

\section{Conclusions}

Magnetic maghemite nanoparticles $\left(\mathrm{Fe}_{2.75} \mathrm{O}_{4}\right)$ were synthesized by laser target evaporation. They had an inverse spinel structure. The size of the coherent scattering region $\mathrm{D}_{\mathrm{XRD}}$ determined by the Scherrer method based on the XRD patterns and the number average diameter calculated from the TEM data correlate well with each other: $D_{\text {XRD }}=23 \pm 3 \mathrm{~nm}$ and $D_{N}=23 \pm 3$ $\mathrm{nm}$. The synthesized MNPs were used to produce electrostatically stabilized aqueous suspensions with 5 $\mathrm{mM}$ sodium citrate. The maximum iron oxide concentration of was $37 \mathrm{~g} / \mathrm{l}$ with an average hydrodynamic aggregate size of $86 \mathrm{~nm}$ and averaged value of the zeta potential $(-42.5) \mathrm{mV}$.

Exophiala nigrum eukaryotes were grown either in the presence or in absence of MNPs. Strain R-11 Exophiala nigrum was isolated from a sample of Baikal water taken from the surface of the layer at a distance of $5 \mathrm{~m}$ from the shore in the Baikalsk area. De-aggregated suspensions of $\mathrm{Fe}_{2.75} \mathrm{O}_{4}$ MNPs were added in concentrations of 1 to $10^{4}$ maximum permissive dose. Cells were exposed for 24 to 96 hours periods and then plated onto a solid medium. Suspensions of MNPs in the concentration range of 1 MPD to 10 MPC did not induce toxic effects. Additions of a MNPs suspension in iron concentrations of 100 and 1000 MPD starting from 72 hours of exposure caused a stimulating effect.
Very pronounced stimulating effect was revealed for a suspension of MNPs at a concentration of 10000 MPD of iron. A significant excess of the number of cells in 1 $\mathrm{ml}$ compared with the control was manifested in the first day of exposure. At exposures of 72 and 96 hours, the difference with the number of cells in the control was 11 and 30 times, respectively $(\mathrm{P}<0.05)$. Suspensions of nanoparticles in concentrations of $100 \mathrm{MPC}$ and $10^{3}$ MPD are moderately toxic, but at a $10^{4}$ MPD they become toxic.

The work was supported by MEC MAT2014-55049-C2-1-R, state work № 0389-2014-0002 and RFBR №16-34-50192 grants. We thank A.P. Safronov, Iu. Novoselova, A.M. Murzakaev and I.S. Tyukova for special support. Selected measurements were made at SGIKER services UPV/EHU.

\section{References}

1. H. Matusiewicz, Acta Biomater.10, 2379 (2014).

2. J.P. Novoselova, A.P. Safronov, O. Samatov, I.V. Beketov, H. Khurshid, Z. Nemati, H. Srikanth, T. P. Denisova, R. Andrade, and G.V. Kurlyandskaya, IEEE Trans. Magn. 50, 4600504 (2014).

3. R. C. O'Handley, Modern Magnetic Materials (John Wiley \& Sons, New York, 1972) p. 740.

4. G.V. Kurlyandskaya, Iu.P. Novoselova, V.V. Schupletsova, R. Andrade, N.A. Dunec, L.S. Litvinova, A.P. Safronov, K.A.Yurova, N.A. Kulesh, A.N. Dzyuman, and I.A. Khlusov 431, 249 (2017).

5. I. S. Lyubutin, C. R. Lin, Yu. V. Korzhetskiy, T. V. Dmitrieva, R. K. Chiang, J. Appl. Phys. 106, 034311 (2009)

6. Y. Hwang, J.-K. Lee, Y.-M. Jeong, S. Cheong, Y.-Ch. Ahn, and S. H. Kim, Powder Technology 186, 145 (2008).

7. J. H. Grossman and S. E. McNeil, Physics Today, 38 (August, 2012).

8. G.V. Kurlyandskaya, E. Fernandez, A.P. Safronov, A.V. Svalov, I.Beketov, A. Burgoa Beitia, A. GarciaArribas, F.A. Blyakhman, Appl. Phys. Lett. 106 193702 (2015).

9. V.V. Osipov, V.V. Platonov, M.A. Uimin, and A.V. Podkin, Tech. Phys. 57, 543 (2012).

10. I.P. Babieva and I.Yu. Chernov, Biology of yeasts (M.V. Lomonosov MSU, Moscow, 2004).

11. A.I.Netrusov, M. A. Egorova, L. M. Zakharchuk et al. Workshop on a microbiology (Moscow, 2005).

12. N. V. Abramov, S. P. Turanska, A. P. Kusyak, A. L. Petranovska, P. P. Gorbyk, J. Nanostruct. Chem. 6, 223 (2016).

13. I.V. Beketov, A.P. Safronov, A.I. Medvedev, A. I. Medvedev, J. Alonso, G. V. Kurlyandskaya, and S. M. Bhagat AIP Adv. 2, 022154 (2012).

14. P. Scherrer, Nachr Ges Wiss G*ottingen 26, 98 (1918).

15. A.P. Safronov, private communication at Ural federal university (2017).

16. N.A. Kulesh, I.P. Novoselova, A.P. Safronov, I.V. Beketov, O.M. Samatov, G.V. Kurlyandskaya, M.V. Morozova, and T.P. Denisova, J. Magn. Magn. Mater. 415, 39 (2016). 